\title{
HAIDINGER'S BRUSHES AND THE RETINAL RECEPTORS* \\ With a Note on the Stiles-Crawford Effect
}

BY

\author{
A. Stanworth and E. J. Naylor
}

Manchester

Haidinger (1844) described an entoptic phenomenon seen in plane polarized white light consisting of the appearance round the fixation point of yellow and blue " brushes " or "sheaves ", the yellow brushes being parallel to the plane of polarization of the light. This appearance was the subject of much speculation during the following years, and several explanations for the phenomenon were advanced; the explanation given by Helmholtz (1856) was generally accepted until it was questioned by Cogan (1941).

\section{Theories formerly advanced}

These are the major theories advanced to explain the appearance :-

I. That the brushes are due to multiple refractions at the boundary surfaces of the intra-ocular media.

II. That they are due to the intra-ocular media acting as doubly-refracting plates.

III. That they are due to radial fibres in the macular region acting as an analyser.

IV. That they are due to subtotal reflection of rays falling obliquely on the cones.

I. This theory, which was put forward by Erlach (1847) and Jamin (1848), was disproved. by Helmholtz (1856), who showed that the brushes should, on this hypothesis, be centred along the optic axis, not the fixation axis, and also that they should become more marked towards the periphery of the retina, which is not the case. A further objection is that the brushes should appear almost equally distinct in all homogeneous colours, whereas they are most distinctly seen in blue light.

II. The theory that the brushes are due to the intra-ocular media acting as doubly refracting plates (Silbermann, 1846; Brewster, 1850) was recently revived by Cogan (1941). It is based on the fact that if a cornea or lens is placed between a polarizer and a crossed analyser, a black " cross" is seen, due to the doubly-refracting properties of the fibres and the structure (Stanworth and Naylor, 1950a). If a second doubly-refracting plate of appropriate retardation (presumably approximating to a wave

\footnotetext{
* Received for publication August 6, 1949.
} 
plate) is then inserted between the polarizer and analyser the appearance can be changed to yellow and blue sectors which may bear a fairly close resemblance to Haidinger's brushes (Cogan, 1941).

Cogan, in his description of this theory, admits that he knows no structure in the eye that could act either as an interference plate or as an analyser. Silbermann (1846) postulated that the cornea and anterior part of the lens acted as the birefringent elements, and that the posterior part of the lens fibres, arranged radially, acted as the analyser, possibly assisted by some regular radial arrangement in the vitreous, whereas Brewster (1850) ascribed the analysing effect to the retina. However, an analyser with radially arranged elements would not produce the appearance of brushes for all positions of the original plane of polarization: what would be needed is an analyser in which all the elements were always crossed with the original plane of polarization, i.e., an analyser which rotated with the rotation of the polarizer. It is difficult to imagine any intra-ocular structure which would do that. Brewster himself also pointed out the further difficulty that the directions of the blue and yellow brushes would always be at $45^{\circ}$ to the plane of polarization of the polarizer and analyser, which is, of course, not the case. Another objection is that the centre of the brushes should appear red, as is shown in Cogan's picture of the interference figure, whereas this is, of course, not seen in the entoptic figure.

We have carried out further experiments which do not support this theory. If a small window is cut in the posterior part of the eyeball without disturbing the vitreous, and polarized light is allowed to enter the cornea and pupil, the emergent light can be observed through this window which is closed by a strain-free glass plate. The pupil then appears to be uniformly illuminated, showing that the cornea, lens, and vitreous do not contain any structures which act as an analyser. On Cogan's hypothesis, therefore, the postulated analyser must be in the retina. If a Nicol prism or piece of polaroid is used to represent this, the appearance shown overleaf in Fig. 1 is seen, projected into the pupillary area. This " cross" is analogous to that seen when the cornea alone is placed between a polarizer and crossed analyser (Stanworth and Naylor, 1950a). Light reaching the macula from that part of the pupillary area occupied by the dark cross must be polarized in its original direction: in addition, owing to the symmetry of the figure, the light from the remaining quadrants will not alter this state of plane polarization to any great extent. The macula will thus be uniformly illuminated by approximately plane polarized light, and will not be capable of appreciating the cross as such.

In addition, if plane polarized white light is passed through a 


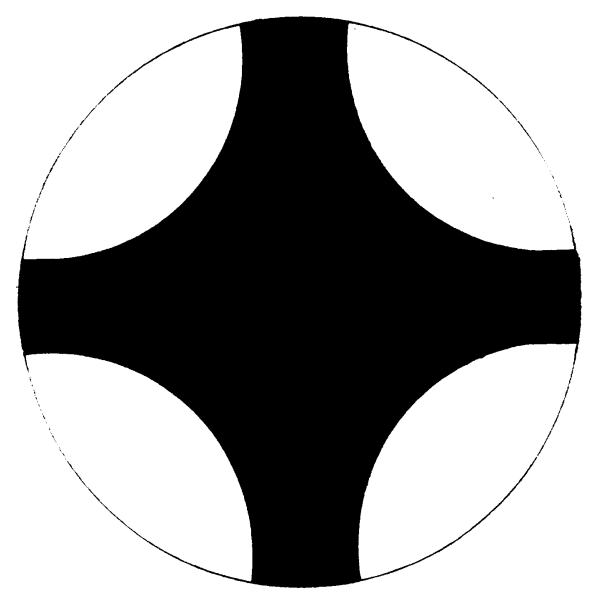

Fig. 1.

quarter-wave plate placed in a diagonal position to the polarizer, and is allowed to fall on a combination of a cornea and interference. plate such as postulated by Cogan, the pattern seen is very different from Haidinger's brushes. It consists essentially of two red dots in opposite quadrants, as in Fig. 2, the arrangement of the other colours depending on the orientation of the quarter-wave plate relative to the interference plate. In practice, no such pattern is

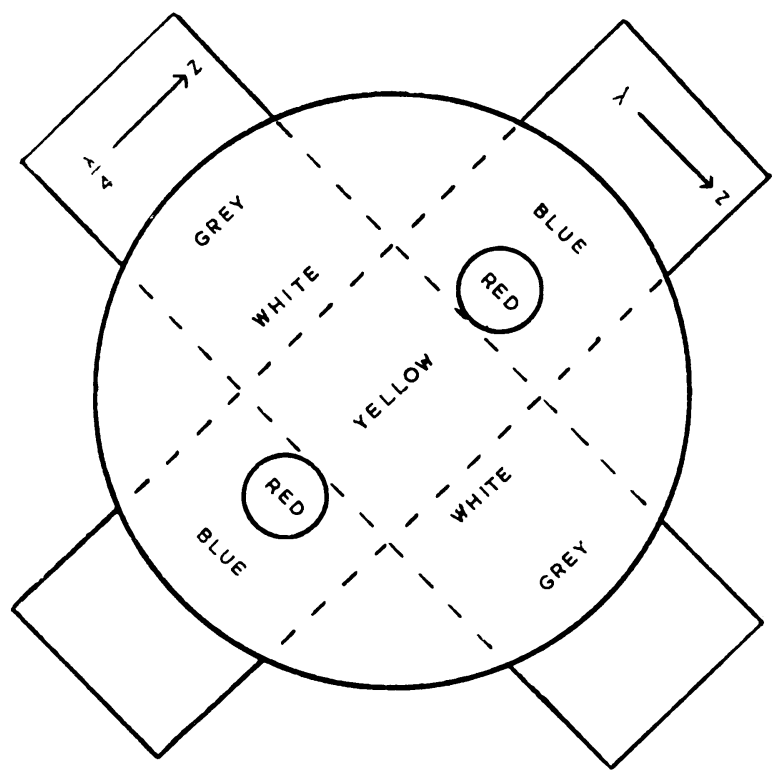

FIG. 2. 
seen entoptically with a quarter-wave plate, the appearance of the brushes remaining substantially unaffected, though they are rather less easily seen. Under the same conditions in monochromatic light (so that circularly polarized light is produced), the appearance on Cogan's hypothesis should be of two dark dots in opposite quadrants. This again is not seen entoptically, for the brushes, if seen at all, retain their original form. In fact, Boehm (1940) has shown that all the entoptic phenomena induced by placing various' interference plates between the polarizer and the eye can be explained by postulating that the brushes are always seen along the major and minor axes of the elliptical vibration produced, provided that the retina acts as a radial analyser and allowance is made for a retardation in the ocular media of about $50 \mathrm{~m} \mu$. The cause of this retardation has been discussed in another communication (Stanworth and Naylor, 1950a).

III. This hypothesis is that the brushes are due to a radial analyser, anterior to the rods and cones, which absorbs blue light to a greater degree when it is polarized in one direction (Helmholtz, 1856). Since the brushes are approximately equal in extent to the projection of macular area, the analysing action is usually taken to be due either to Henle's fibres or to Muller's fibres, both of which are radially arranged in this area. In order to explain the yellow colour of the darker brushes, Helmholtz assumed that in some manner the analyser strongly absorbs the extraordinary vibration for blue light (i.e., it is dichroic). This would result in a sensation of yellow in the meridian in which this absorption is at a maximum. 'This blue-absorbing substance is often assumed to be the yellow pigment of the macula. The cause of the blue brushes is rarely discussed, but is usually taken to be due to contrast (Stokes, 1850).

To this theory several objections may be made :

(a) The yellow pigment is not associated with either Henle's fibres or Muller's fibres. In fact, it is distributed throughout the cerebral layers (Dimmer, 1907) and so is less plentiful in the fovea than in the peri-foveal region. On Helmholtz's theory the yellow brushes should, therefore, be either absent or much diminished in intensity at the fixation point, and in blue light the centre of the brushes should be light in colour. In fact, the centre is definitely seen to be dark, as are the originally yellow brushes.

(b) Though all nerve fibres are birefringent, there is no evidence that any of the radial elements of the macula are dichroic. Boehm (1940), though he supports Helmholtz's theory, states that if the isolated retina including the macular region is examined in polarized light, no brushes can be seen objectively, though they should be visible if the radial fibres of the macula are dichroic. 
(c) On Helmholtz's hypothesis, the maximum absorption for blue would occur in the meridian parallel to the plane of polarization, and there would be a gradual decrease in the percentage absorption as the absorbing elements are more and more inclined to this meridian. Only in the meridian at right angles to the plane of polarization would no absorption occur, and so in all meridians except this, there should be an appearance of a yellow colour of varying intensity. In the model of a dichroic structure made by Boehm to correspond as far as possible with the radial fibres of the macula, yellow brushes are produced in white light, and dark grey brushes in blue light, but apparently nothing is seen corresponding to the blue or light sectors respectively. The uniformity in colour of the yellow brushes, and the existence of blue brushes, is thus unexplained.

(d) The blue brushes are not adequately explained by contrast. Their blue colour is not more marked at its border with the yellow, and their intensity is greater than would be expected from a contrast phenomenon. In addition, an object giving an image of the same size and colour as the yellow brushes does not produce such a contrast effect. We have also found that a negative afterimage of the brushes is easily obtained, in which the positions of the brushes appear to be reversed. The blue brushes produce an after-image which is at least as vivid as that produced by the yellow brushes, which would not be expected if the blue brushes were merely contrast effects.

(e) We have also found that after five minutes' adaptation to blue light, the first brushes to appear are the blue ones, which would never occur if the only primary phenomenon was that producing the yellow brushes.

$(f)$ It is believed by some observers that the yellow pigment of the macula is not present during life (Gullstrand, 1906). However, this objection is probably not valid, as the pigment can be seen by ophthalmoscopy, using almost, but not completely, red-free light (Thorner, 1932). If the macula is examined in red-free polarized light, one might expect on Helmholtz's theory that the yellow appearance would be most marked in the meridian parallel to the plane of polarization. Unfortunately, under these conditions, we have not been able to see the macula sufficiently distinctly to reach a definite conclusion.

(g) Gullstrand (1906) found that he could not obtain parallax between the peripheral parts of the brushes and the macula when using a pinhole, and concluded from this that the site of origin of the brushes must be in the sensory epithelium layer, and not in Muller's fibre layer.

IV. As we have seen, Gullstrand thought the origin of the brushes to be in the Tayer of sensory epithelium: he postulated 
that it was connected with loss of light due to subtotal reflection occurring in those rays falling obliquely on the cones. The amount of this loss would be affected both by the plane of polarization and by the wavelength of the incident light.

One objection to this theory is that Barany (1946) has, as we shall see later, failed to find any such influence of the plane of polarization in the sensation of brightness from oblique rays falling on the retina. In addition, though the details of Gullstrand's theory are not very well worked out, this hypothesis, like that of Helmholtz, leads to the deduction that the brushes would appear to be of one colour only, the brightness alone varying in different meridians. The colours observed, therefore, are not explained.

\section{New Theory}

It woùld appear, then, that previous theories are scarcely satisfactory. Any theory must, to be adequate, explain the existence of the blue brushes as a positive phenomenon, and should fit in with modern knowledge of the birefringence and dichroism of the retina. The following points are of importance :-

(1) In the retina, the rods, cones, and nerve fibres are birefringent, but the only strongly dichroic structures are the unbleached rods (Schmidt, 1935).

(2) The blue receptors may be rods, or something intermediate between rods and cones (Willmer, 1946; Granit, 1947).

(3) It is the electric vector which is usually considered to be responsible for initiating photochemical changes (Houston, 1938).

(4) The light reaching the macula is, as we have already shown, substantially polarized in its original direction.

In view of these factors, it is suggested that the structures concerned in analysing the light falling on the retina are the blue receptors, which could be dichroic owing to a regular arrangement of the molecules of the "blue substance," similar to that of the molecules of the visual purple in the dichroic rods. It is postulated that this dicroic pigment is so united with the structure of the receptors that the latter are in some way orientated radially in the macula. The blue receptors which are lying in the direction of the electric vector would then strongly absorb the incident blue light. This absorption would be greater than the average absorption by the randomly arranged blue receptors in the peripheral field: consequently a sensation of blue would be produced. The light would, however, be only weakly absorbed by the blue receptors lying perpendicular to the electric vector, and consequently the preponderance of stimulation of the remaining retinal receptors would result in the complementary sensation of yellow. The fact that both the yellow and blue brushes are positive phenomena is thereby explained. On this theory as it stands, the mid-lines of 
the yellow and blue brushes should present the deepest colour, which should fade away to white at the boundary between the brushes. At this boundary, however, simultaneous contrast would be expected to reinforce the colour on either side, and might well produce the uniform yellow and blue brushes seen.

This hypothesis has thus many advantages over that of Helmholtz; there are, however, two possible objections :

(1) The fovea itself may be blue-blind (Willmer and Wright, 1945) and consequently unable to take part in these suggested

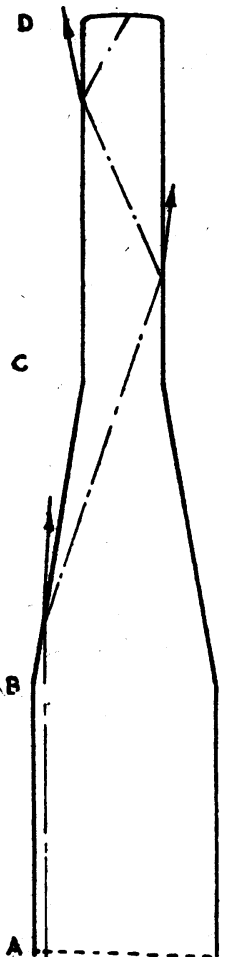

FIG. 3. phenomena. It may well be, however; that the central blue-blind area, which in normal use of the eyes is not seen as such (owing, possibly, to physiological filling-in of the blue scotoma and the slight ocular movements) will be perceived when it is flanked closely on each side by a yellow sensation. In this case the yellow sensation would include the fovea, as is the case with the brushes.

(2) For light passing along their axes, the unbleached rods are not dichroic, but absorb light polarized in all meridians (Schmidt, 1935). In order to exhibit dichroism, the light must pass across the length of the rod and, presumably, of the blue receptors also. It must be remembered, however, that in all probability light falling normally on the retina does not pass directly along the axis of the receptor. A hypothetical diagram of the structure of a cone and of the pathway of light through it, similar to that given by O'Brien (1947) is shown in Fig. 3, where it will be seen that light is internally reflected in the cone ellipsoid BC and passes obliquely, with several more internal reflections, through the outer member $\mathrm{CD}$; during this passage the postulated dichroic action could take effect.

The fact that the light from the peripheral part of the pupil must fall obliquely on the receptors gives rise to the idea of a second possible mechanism whereby the dichroic action could occur. In this case it might be expected that the brushes would be seen more clearly with a dilated pupil, but, if the mechanism described above is adequate, the extra absorption from the increased obliquity might be inappreciable. Also the larger effective birefringence of the more peripheral parts 
of the cornea and lens would tend to obscure the simple polarization of the light falling on the retina and so to decrease the clarity of the brushes. In fact, we have been unable to be certain of any alteration in clarity with a dilated pupil.

One further point of interest is that the rate of adaptation of the blue receptors may be greater than that of the other receptors (Wright; 1934; Granit, 1947). In view of this, after adaptation to white light, it might be expected that the blue brushes would appear first. Unfortunately, the speed at which the brushes appear under these conditions is so rapid that it is difficult to be certain whether or not this is so.

\section{Summary}

1. Previous explanations of Haidinger's brushes are discussed.

2. A new theory is advanced that they are. due to postulated dichroic properties of the blue receptors of the macula.

\section{A NOTE ON THE STILES-CRAWFORD EFFECT}

The directional sensitivity of the retina described by Stiles and Crawford (1933) in which the sensation of brightnessoproduced by light entering the periphery of the pupil is much less than that produced by the same light entering centrally, was ascribed by Wright and Nelson (1936) to the transition from sub-total reflection to total reflection in the light-percipient elements of the retina in these circumstances. Barany (1946) attempted to test this theory by using polarized light, on the basis that in sub-total reflection the relationship between the energy of the refracted and reflected rays depends on the plane of polarization of the incident light, and hence, on the theory of Wright and Nelson, the intensity of stimulation should also depend on the plane of polarization. He could not find any such variation, however, and concluded that his negative results argue against Wright, and Nelson's theory, though they do not definitely disprove it, since it is not known how large the anticipated effect would be if the theory were correct. The theoretical basis of the experiment was also further discussed by O'Brien $(1946 ; 1947)$ and Platt $(1947)$.

In these discussions, however, no account was taken of the effect produced upon the plane of polarization by the birefringence of the cornea and lens. We have already shown in the discussion of Haidinger's brushes that if the whole pupillary area is illuminated the light reaching the macula is still substantially polarized in its original direction. If, on the other hand, in an eye with the posterior scleral window described above, a thin pencil of plane polarized light is allowed to enter the pupillary area at a point 
which is not situated on the interference figure, it will be found that the crossed analyser does not completely extinguish the emergent light, showing that the plane of polarization has not remained unchanged. For a pencil of light entering the pupil at any point on the interference figure (which includes the centre of the pupil) the light is almost completely extinguished by such an analyser and therefore the plane of polarization has remained substantially unaltered. If the polarizer and analyser are rotated together the interference figure is seen to rotate with them, and any point near the periphery of the pupillary area lies on the interference figure four times in each complete revolution.

Under the conditions of Barany's experiment, in which the polarizer is rotated at a speed of 2-10 revolutions per second, the light reaching the retina should, therefore, be polarized in its original direction four times per revolution. For all other directions of the polarizer its state of polarization will depend on the magnitude of the birefringence of the cornea and lens, which will lead, in general, to some form of elliptical polarization.

It is impossible to calculate with any accuracy the effect that this elliptically polarized light will have on the quantity of light lost by sub-total reflection, since the conditions both of the light itself and of the retinal structures are not known with any certainty. It can be said, however, that the expected variation in the sensation of brightness produced by the type of variable polarization described above will never be greater than that postulated by Barany on the original supposition that the light reaches the retina plane polarized; in fact, it is most likely that it will be less.

It appears then that consideration of the effects of the birefringence of the ocular media supports the view that the negative results obtained by Barany do not exclude the explanation of the Stiles-Crawford effect advanced by Wright and Nelson.

\section{REFERENCES}

Barany, E. H. (1946). Acta ophthal. Kbh., 24, 93.

BОЕнм, G. (1940). Ibid., 18, 109.

Brewster, D. (1850). Rep. Brit. Ass., 2, 5.

Cogan, D. G. (1941). Arch. Ophthal., Chicago, 25, 391.

Dimmer, F. (1907). Graefes Arch. Ophthal., 65, 486

Erlach, K. von (1847). Arch. Anat. Physiol., 313.

Granit, R. (1947). "Sensory Mechanisms of the Retina." Pp. 284 and 341. Oxford Univ. Press, London.

Gullstrand, A. (1906). Graefes Arch. Ophthal., 62, 1.

Haidinger, W. (1884). Ann. Phys. Chem., Berlin, 63, 29.

Helmhol $z$, H. (1856-66). "Handbuch der physiologischen Optik". Amer. ed. (1924) 2, p. 304 fol.

Houston, R. A. (1938). "'Treatise on Light". 7th ed. p. 458. Longmans, Green, London

Jamin, J. (1849). Ann. Phys. Chem., Berlin, 74, 145. 
O'Brien, B. (1946). J.opt. Soc. Amer., 36, 506. (1947). Ibid., 37, 275.

Platt, J. R. (1947). Ibid., 37, 669.

SCHMIDT, W. J. (1935). Z. Zellforsch., 22, 485.

Silbermann, J. T. (1846). C. R. Acad. Sci., Paris, 23, 629.

StanWORTh, A. and NAYLOR, E. J. (1950). British Journal of Ophthalmology, 34, 201.

Stiles, W. S. and Crawford, B. H. (1933). Proc. roy. Soc. B, 112, 428.

Stokes, G. G. (1850). Rep. Brit. Ass., 2, 20.

THORNER, W. (1932). Brit. J.physiol. Optics, 6, 16.

Willmer. E. N. (1946). "Retinal : tructure and Colour Vision," p. 150. Cambridge Univ. Press, London. and WRIGHT, W. D. (1945). Nature, Lond., 156, 119.

Wright, W. D. (1934). Proc. roy. Soc. B, 115, 49. and Nel.son, J. H. (1936). Proc. phys. Soc., 48, 401. 